\title{
GESCHÄFTSPROZESSE
}

\section{Verfahren strukturieren, Abläufe optimieren}

VON MATTHIAS KONRAD UND BERND CONRAD

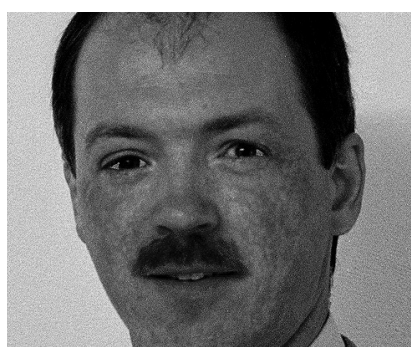

Matthias Konrad ist Regionalleiter Nord des Beratungsunternehmens contec $\mathrm{GmbH}$.

Internet http://www.contec.de

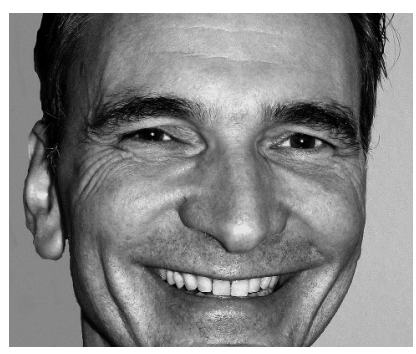

Bernd Conrad ist Geschäftsführer der Lebenshilfe für Menschen mit Behinderung, Kreisvereinigung Detmold e. $\mathrm{V}$.

Internet http://www.

lebenshilfe-detmold.de

\author{
Mit systematischer Prozessoptimierung können Struk- \\ turen und Anläufe effektiv gestaltet werden. Dabei geht \\ es letztendlich immer um bessere Leistungen für die \\ Nutzer und um einen schonenderen Einsatz knapper \\ Ressourcen, wie ein Praxisbeispiel aus der Behinderten- \\ hilfe zeigt.
}

Diskussionen über die angespannte wirtschaftliche Situation der öffentlichen Haushalte und die Auswirkungen auf die Sozialwirtschaft kann man bei vielen Veranstaltungen mitverfolgen, auf denen sich Leitungskräfte aus sozialen Einrichtungen begegnen. Aber auch die Wirtschaftskrise selbst ist in vielen sozialen Unternehmen angekommen, insbesondere in den Werkstätten für behinderte Menschen. Immer wieder wird in Beratungsprojekten davon berichtet, dass es schwieriger wird, Fertigungsaufträge zu akquirieren. Vor diesem Hintergrund wird es zunehmend wichtig, die Strukturen und Abläufe in sozialen Einrichtungen im Sinne der Effektivität und Effizienz zu verbessern.

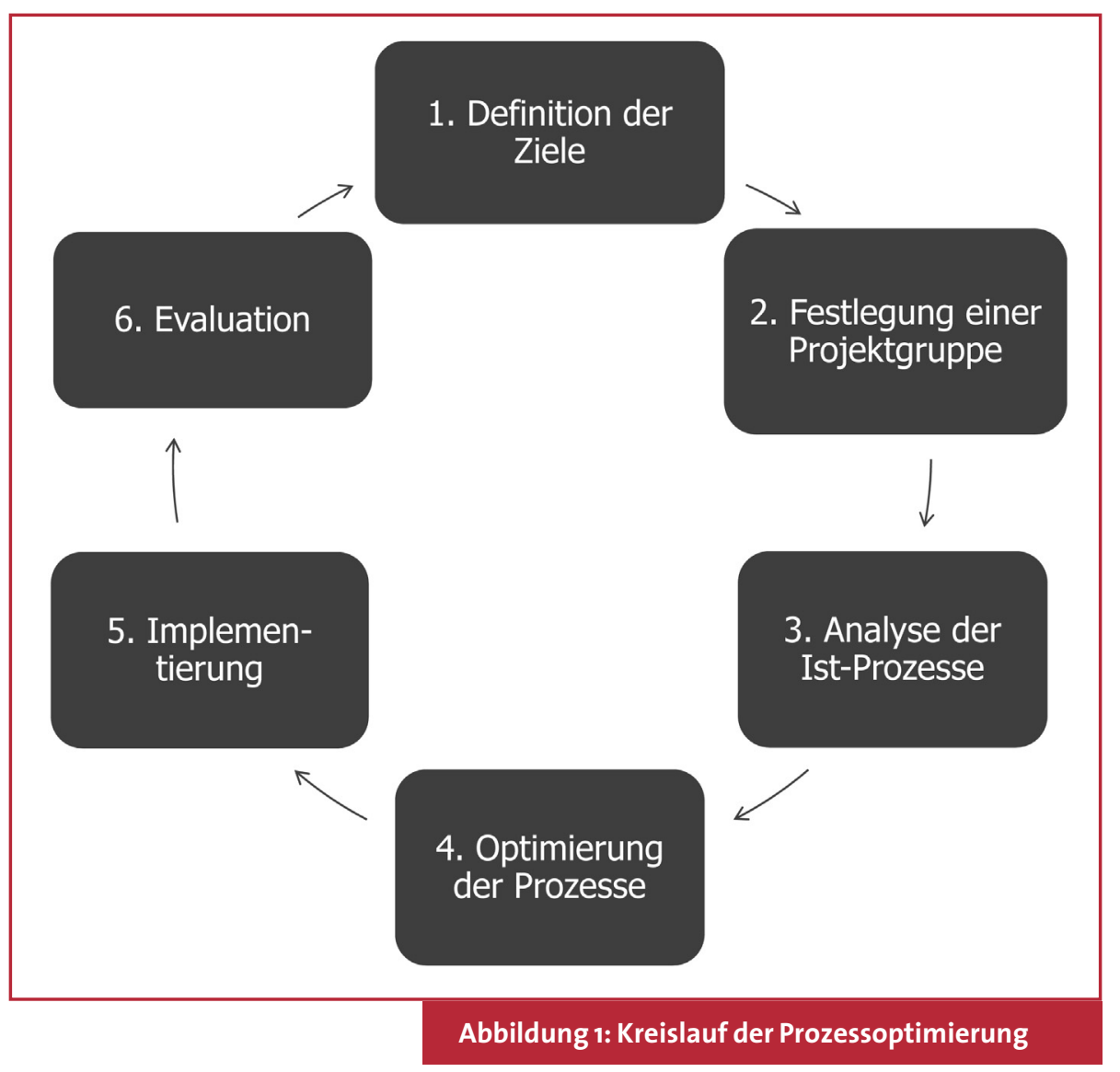


Eine Möglichkeit, dies zu tun, ist die systematische Analyse und Optimierung von Prozessen im Rahmen eines Qualitätsmanagements bis hin zur Zertifizierung nach einer externen Norm. Auf diesem Weg können aktuelle Strukturen vor dem Hintergrund der Kundenanforderungen und Prozesse überprüft werden. Die Einheitlichkeit der Prozessabläufe wird verbessert und das Wissen hierüber gesichert, beispielsweise im Falle eines Mitarbeiterwechsels. Schließlich fördert die Einbindung der Mitarbeiter in den Optimierungsprozess die Motivation, die Arbeitszufriedenheit und die Verbindlichkeit der Arbeitsergebnisse.

»Die letzte Definition und Vereinheitlichung von Prozessen ist in der Lebenshilfe Detmold schon einige Jahre her. Seit dem hat sich vieles verändert. Wir wollten im Rahmen eines Optimierungsprozesses klären, an welchen Stellen unsere Arbeit besser an den Anforderungen der Eingliederung in den allgemeinen Arbeitsmarkt ausgerichtet werden kann. Über die Stärkung eines einheitlichen Verständnisses für Prozesse und die mit ihnen zu erreichenden Ziele sollte letztlich auch der Einsatz an Ressourcen und damit im Wesentlichen der Arbeitszeit verbessert werden. In diesem Prozess einen Blick von außen einzubinden, hilft uns bei der Überwindung alter Gewohnheiten und sichert auch Lerneffekte aus den Erfahrungen an anderer Stelle. « - Bernd Conrad, Geschäftsführer Lebenshilfe Detmold e. V.

\section{Wie man Prozesse in sechs Schritten optimiert}

Da sich die Anforderungen der Kunden an die Leistungen einer Einrichtung immer wieder ändern, sollte eine Prozessoptimierung als sich regelmäßig wiederholender Kreislauf etabliert werden. Dieser Kreislauf besteht erfahrungsgemäß aus sechs Schritten: Definition der Ziele, Festlegung der Projektgruppe, Analyse der Ist-Prozesse, Optimierung der Prozesse, Implementierung, Evaluation (vgl. Abbildung 1, Seite 7).

\section{Schritt: Definition der Ziele}

Die Zielsetzungen für eine Prozessoptimierung können unterschiedlich sein.

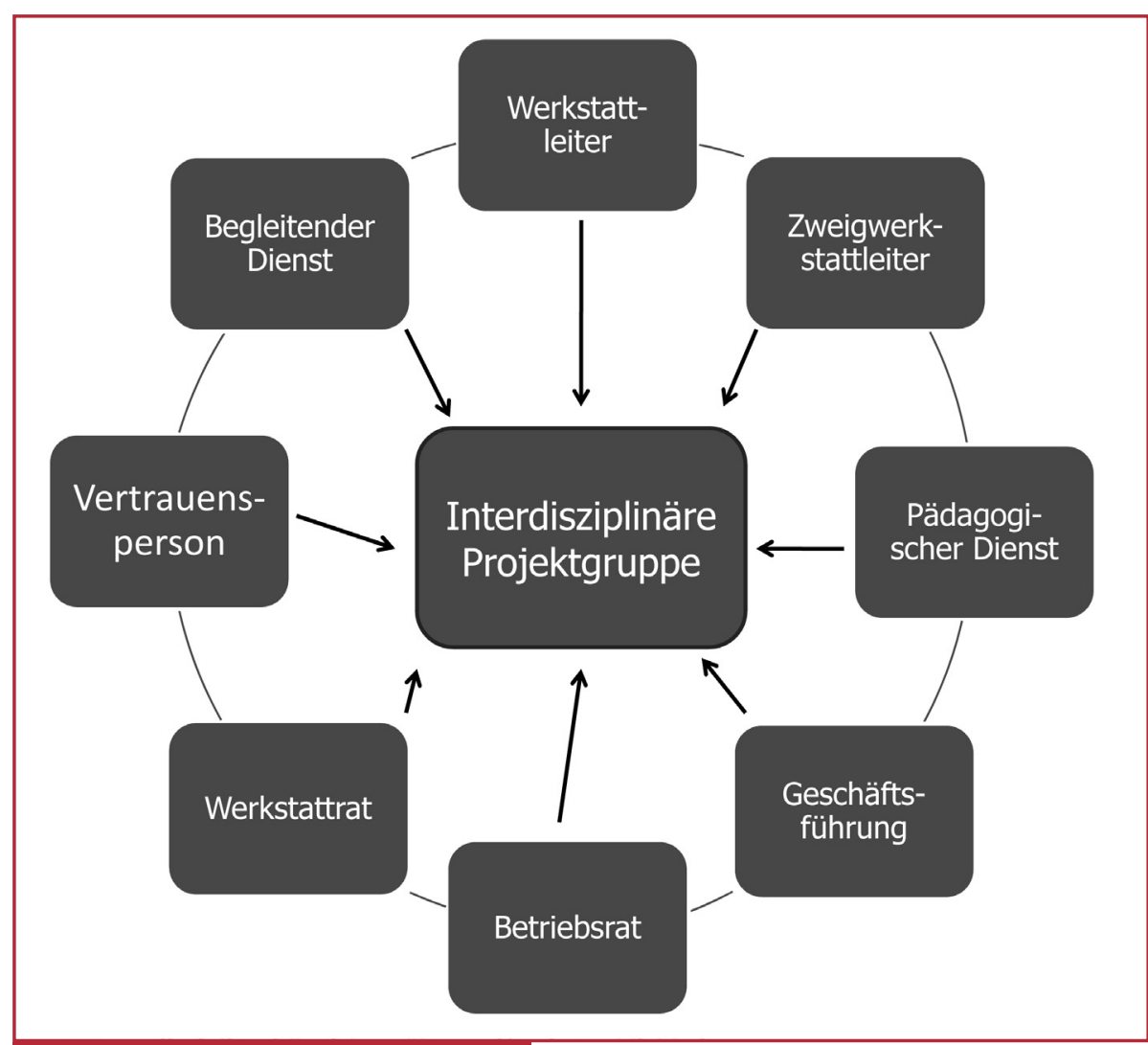

Abbildung 2: Interdisziplinarität

sichert übergreifende Fachlichkeit

Damit alle an der Optimierung Beteiligten ihr Engagement gleich ausrichten können, ist es wichtig, diese Ziele am Anfang des Prozesses festzulegen und zu kommunizieren. Oder besser noch: gemeinsam mit den Beteiligten zu erarbeiten. Ziele der Prozessoptimierung können sein:

- Es besteht ein gemeinsames Verständnis für die bestehenden Prozesse.

- Es liegt ein gemeinsames Verständnis über die Bedeutung von wesentlichen Rahmenbedingungen vor (z. B. Normalität für Menschen mit Behinderungen, Beteiligung von Menschen mit Behinderungen etc.).

- Die Schnittstellen sind transparent.

- Reibungsverluste sind minimiert.

- Ein Standard zur Verbesserung von Effizienz und Effektivität ist entwickelt.

- Die Zusammenarbeit zwischen unterschiedlichen Professionen und Abteilungen ist verbessert.

- Für neue Mitarbeiter liegen übersichtliche Prozesse vor, die die Einarbeitung erleichtern.

- Es sind Optimierungsansätze für den Einsatz der EDV ermittelt.
Letztendlich geht es bei einer Prozessoptimierung immer um die Verbesserung der Leistungen im Sinne der Qualität oder des Ressourceneinsatzes.

»Für die Projektgruppe in der Lebenshilfe Detmold gab es unterschiedliche Erwartungen und Ziele. Sie begannen bei gesteigerter Übersichtlichkeit der Abläufe, führten über Verdeutlichung der wichtigen Prozesse in allen Bereichen bis hin zu einer Verbesserung des einheitlichen Verständnisses zu den Prozessen im Sinne einer Förderung der Eigenständigkeit der Mitarbeiter und ihre Eingliederung in den allgemeinen Arbeitsmarkt und auch einer Arbeitserleichterung für alle Beteiligten. Darüber hinaus sollen die optimierten Prozesse eine erste Grundlage für die Zertifizierung dieses Bereiches sein."Bernd Conrad, Geschäftsführer Lebenshilfe Detmold e. V.

\section{Schritt: Festlegung der Projektgruppe}

Die Zusammenstellung der Mitglieder einer entsprechenden Projektgruppe ist im Wesentlichen abhängig da- 


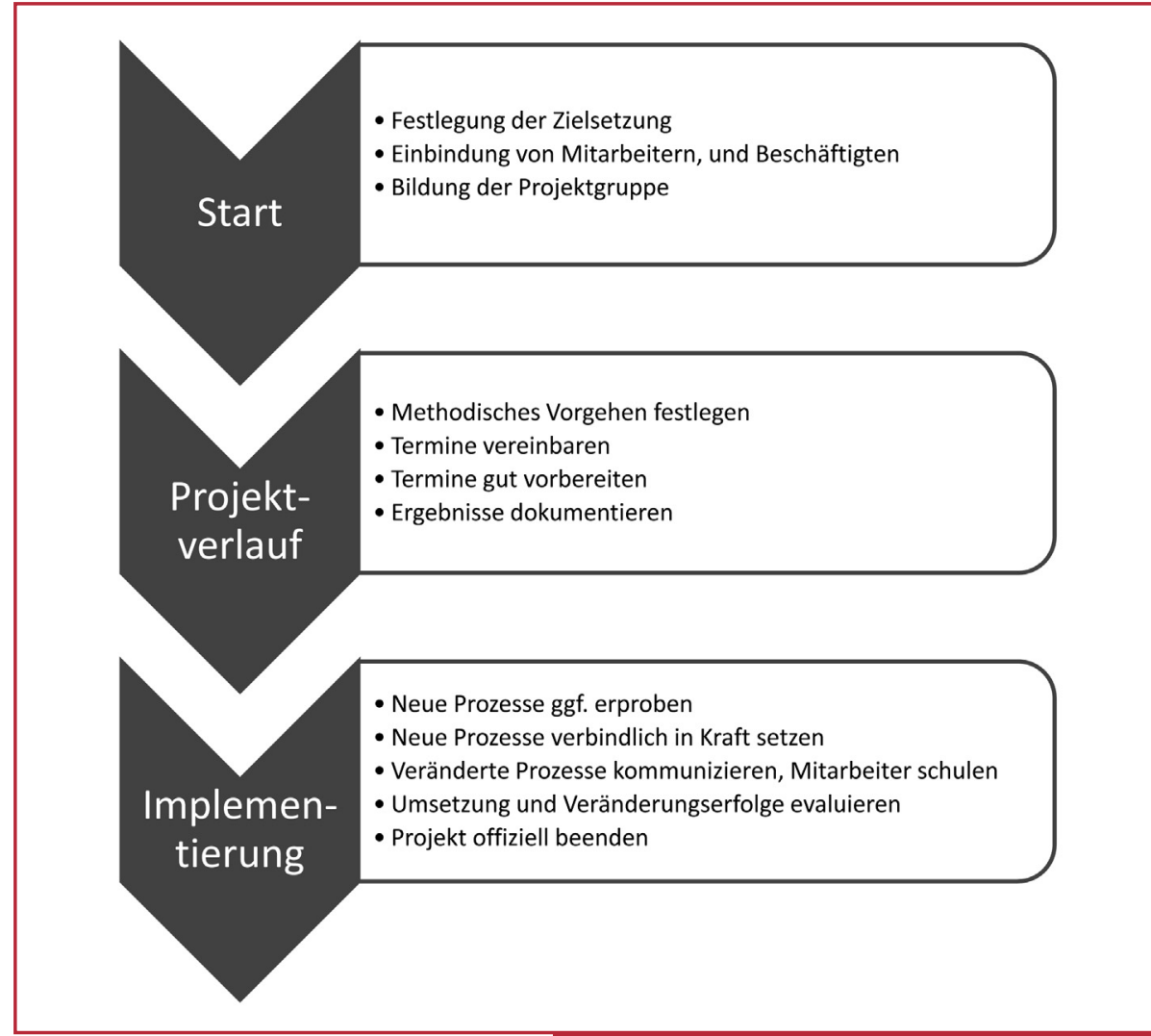

Abbildung 3: Schritte im Projektverlauf

von, welche und wie viele Personen in welcher Funktion die Umsetzung der Zielsetzung beeinflussen können. Dementsprechend sollten alle notwendigen Personen beteiligt werden, ohne aber die Arbeitsfähigkeit der Gruppe zu gefährden. So besteht auch in den Werkstätten der Lebenshilfe Detmold die Projektgruppe aus den Führungsund Fachkräften mit den maßgeblichen Steuerungsfunktionen (vgl. Abbildung 2 .

Checkliste Prozessoptimierung

Worauf man achten sollte:

$\checkmark$ Positive Lernkultur etablieren (Bestehendes nicht schlecht machen, sondern Potentiale für die Zukunft suchen)

$\checkmark \quad$ Festlegung der Ziele für die Prozessoptimierung

$\checkmark$ Einbindung Betriebsrat / Mitarbeitervertretung

$\checkmark$ Erwartungen und persönliche Betroffenheiten der Mitarbeiter und Beschäftigten / Bewohner /Klienten einbinden

$\checkmark$ Feste Projektgruppe einrichten

$\checkmark$ Interdisziplinäre Zusammenarbeit sicherstellen

$\checkmark$ Verbindliche Termine vereinbaren

$\checkmark$ Projekttreffen vorbereiten lassen und die Unterlagen vorher an alle Teilnehmer verschicken

$\checkmark$ Möglichst Qualität vor Menge und Schnelligkeit

$\checkmark$ Nachhaltige Umsetzung der Ergebnisse sicherstellen und evaluieren

»In unsere Projektgruppe sind die Werkstattleitung, die Leitungen des pädagogischen und begleitenden Dienstes sowie die Zweigwerkstattleitungen eingebunden. Zusätzlich werden die Interessen aller Mitarbeiter und Beschäftigten durch die Einbindung des Betriebsrates sowie des Werkstattrates sichergestellt. «-Bernd Conrad, Geschäftsführer Lebenshilfe Detmold e. V.

In einer Zwischenevaluation des Projektes wurde deutlich, dass diese Zusammensetzung auch in der Bewertung der Beteiligten als bereichernd wahrgenommen wird. Die Vorteile laut Aussagen der Fachkräfte sind insbesondere:

- Die unterschiedlichen Berufshintergründe machen das Vorgehen interessant und vielfältig.

- Die Gruppenzusammensetzung ist in dieser Form unbedingt notwendig, um Doppelerläuterungen und Doppelstrukturen zu vermeiden.

- Zudem ist es gut, sich von Beginn an mit eigenen Themen einbringen $\mathrm{zu}$ können. Auch der Werkstattrat erlebt die Zusammensetzung als gut, da alle Bereiche der Werkstatt vertreten sind. Darüber hinaus ist ihm wichtig, von der Vertrauensperson in diesem Prozess begleitet zu werden, damit eine Reflexion der Treffen stattfinden kann.

\section{Schritt: Analyse der Ist-Prozesse}

Als Analyseinstrumente der bestehenden Prozesse bieten sich unterschiedliche Methoden an. Soweit die Prozesse dokumentiert sind, kann ein Abgleich zwischen diesem Stand und dem aktuellen Vorgehen sinnvoll sein. Insbesondere dann, wenn die Dokumentation schon alt ist und stark von der aktuellen Praxis abweicht, kann es aber besser sein, die alten Dokumente aus der Betrachtung herauszulassen und die Analyse ausschließlich auf dem Ist-Stand aufzubauen.

Weitere Instrumente, wie beispielsweise Interviews oder Workshops in unterschiedlichen Gruppenzusammensetzungen, sind zur Beteiligung der betroffenen Fachkräfte und Mitarbeiter und zur Vertiefung der Analyse wichtig. 
»In Detmold analysieren wir die bestehenden Dokumente der letzten Jahre gemeinsam in der Projektgruppe. Dies hat den Vorteil, dass wir die vorhandene Zeit und die Ressourcen in einem Schritt nutzen können und zusätzliche Abspracheschleifen zwischen den Beteiligten wegfallen. Dort, wo ein größerer Überarbeitungs- und Optimierungsbedarf erkennbar ist, werden Überarbeitungsentwürfe als Vorbereitungen der Besprechungen in der Gesamtrunde erstellt. « - Bernd Conrad, Geschäftsführer Lebenshilfe Detmold e. V.

\section{Schritt: Optimierung der Prozesse}

Die Optimierung der Prozesse kann wie in Detmold erfolgen, jedoch auch als eigenständiger Arbeitsschritt umgesetzt werden. Im Hinblick auf Aufgabentransparenz, Aufgabenbildung, Automatisierung von Arbeitsschritten und das Informations- und Kommunikationssystem wird erarbeitet, was verbessert werden kann. Hier ist es wichtig, dass die Fachkräfte und Mitarbeitenden über die genannten Instru- mente beteiligt werden. Sie als Experten vor Ort wissen am besten, welche Schnittstellen und Optimierungspotenziale es für jeden einzelnen Prozess gibt.

»Die Optimierung der Prozesse in Detmold verdeutlicht in der Diskussion und Auseinandersetzung mit der Thematik Klarheiten über Zuständigkeiten, Aufgaben und Abläufe. Es wird bewusst, wer wofür verantwortlich ist und dabei direkt überprüft, ob dies ein sinnvoller und praxistauglicher Ablauf ist. Besonders deutlich wurde dies beispielsweise in der Akquise von neuen Aufträgen für die Werkstätten. Hier war zum einen unklar, wer für die Akquise verantwortlich ist, zum anderen, wer in welcher Hinsicht an der konkreten Schaffung von neuen Arbeitsplätzen beteiligt ist. « Bernd Conrad, Geschäftsführer Lebenshilfe Detmold e. V.

Insgesamt wurden bereits Vereinfachungen umgesetzt, die nicht nur den Arbeitsaufwand für die Mitarbeiter, sondern auch für die Fachkräfte reduzieren. Dies wurde vom Werkstattrat sehr positiv bewertet.

\section{Schritt: Implementierung}

Die Umsetzung der überarbeiteten Prozesse sollte mit einem fixen Termin für die Gültigkeit der neuen Prozesse hinterlegt werden. Mit der Optimierung von Prozessen hängen teilweise veränderte Handlungen und Arbeitsschritte von Fachkräften zusammen (vgl. Abbildung 3). Durch einen fixen Termin erhält die Implementierung einen offiziellen Charakter und dient als Startschuss für möglicherweise veränderte Arbeitsschritte.

»Bei uns wird jeder Prozess im gemeinsamen Arbeitskreis verabschiedet und tritt ab diesem Zeitpunkt in Kraft. Über interne Fortbildungstage wurden die Fachkräfte geschult und konnten weitere Anregungen und Verbesserungsvorschläge einbringen. Diese gingen bei der Projektgruppe ein, die über die weitere Bearbeitung entschied. Für die weiteren Prozesse, die erst danach in der Projektgruppe bearbeitet werden, gibt es pädagogische Arbeitskreise. Hier werden die Prozesse besprochen und es können Weiterentwicklungsvorschläge an die Projekt-

\section{Was versteht man eigentlich unter Prozessmanagement?}

Unter Prozessmanagement werden alle planerischen, organisatorischen und kontrollierenden Maßnahmen zur zielgerichteten Steuerung einer Wertschöpfungskette im Unternehmen verstanden, um so die vorgegebenen Ziele bezogen auf die Faktoren Kosten, Zeit, Qualität, Kundenzufriedenheit möglichst optimal zu erreichen. Im Gegensatz zur traditionellen vertikalen Organisation besteht der Grundgedanke des Prozessmanagements darin, die Unternehmen horizontal nach kundenorientierten Prozessen zu gliedern. Ziel ist es dabei, durchgängige Dienstleistungsprozesse mit möglichst wenigen Schnittstellen zu gestalten. Die Unternehmen werden nicht mehr vertikal nach Funktionen oder Produkten gegliedert, sondern horizontal nach Prozessen. Im Idealfall entsteht so eine kundenorientierte Dienstleistung aus einer Hand, gesteuert durch einzelne Mitarbeiter oder Gruppen, die gegenüber den internen oder externen Kunden die volle Verantwortung für die Auftragserfüllung haben. Unterstützt werden diese Prozesse durch moderne Informationstechnologien, die durch informationelle Vernetzung eine grundlegend neue Gestaltung der Dienstleistungsprozesse ermöglichen. Die Prozessorganisation besteht aus den drei Elementen Kernprozesse, Supportprozesse und Kompetenzzentren. Kernprozesse bündeln zusammenhängende Aktivitäten, Entscheidungen und Informationen in einem Verantwortungsbereich ohne interne Schnittstellen. Supportpro- zesse haben unterstützende Aufgaben, damit die Kernprozesse reibungslose ablaufen können. Sie können auch unternehmensextern ausgegliedert werden (Outsourcing). Kompetenzzentren bündeln Spezialisierungswissen und bieten spezifische Kenntnisse an, die in den Kern- und Supportprozessen nicht ausreichend vorhanden sind (z.B. Rechtsfragen, Marketing etc.). Sie haben primär die Dienstleistungsfunktion der Vermittlung von Wissen an die Prozesse. Darüber hinaus müssen sie ständig neues Fachwissen erwerben. Im Unterschied zu den Kernprozessen haben sie keinen direkten Kundenkontakt.

Bernd Maelicke

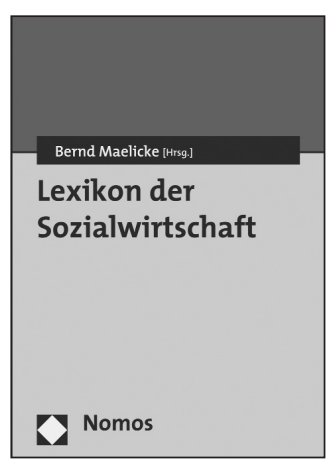

Prof. Dr. Bernd Maelicke ist Direktor des Deutschen Instituts für Sozialwirtschaft und Honorarprofessor an der Leuphana-Universität Lüneburg.

Quelle: Bernd Maelicke (Hg.): Lexikon der Sozialwirtschaft. Nomos Verlagsgesellschaft, Baden-Baden 2007. 1.128 Seiten. 98,- Euro. ISBN 978-38329-2511-6. Seite 8og f. 
gruppe gegeben werden. Danach sind die Prozesse offiziell verabschiedet und treten in Kraft. Die Mitarbeiterinnen und Mitarbeiter in den Werkstätten erhalten die Informationen über veränderte Prozesse und Arbeitsschritte über den Werkstattrat und über die Gruppenleitungen. « - Bernd Conrad, Geschäftsführer Lebenshilfe Detmold e. V.

\section{Fazit}

Insgesamt hat sich das beschriebene Vorgehen zur Prozessoptimierung bereits in vielen Projekten, gerade auch in Detmold, bewährt. Die in dem Detmolder Projekt gewonnenen Erfahrungen, können durch andere Unternehmen der Sozialwirtschaft mit Gewinn genutzt werden (vgl. Abbildung 4, Seite 9).

\section{"Überarbeitete Prozesse müssen mit einem fixen Termin für ihre}

\section{Gültigkeit versehen werden"}

Auch von den Fachkräften kommen bereits positive Rückmeldungen: Insgesamt zeigen sich bei den bis jetzt implementierten und geschulten Prozessen tatsächliche Verbesserungen. Die Verantwortung für die Durchführung der Tätigkeiten ist im Allgemeinen höher, Termine werden verstärkt eingehalten und Regelungen sind verbindlicher.

\section{Schritt: Evaluation}

Die überarbeiteten Prozesse sollten regelmäßig in der praktischen Durchführung evaluiert werden, um die Organisation systematisch weiterzuentwickeln. Prozessuale Potentiale zur Steigerung der Effizienz und Effektivität können damit genutzt werden. Die informelle Weiterentwicklung des Unternehmens wird gesichert und es findet aktives Wissensmanagement in der Organisation statt.

»Die Evaluation der Prozesse soll bei uns in den Werkstätten permanent stattfinden und je nachdem, ob bereichsübergreifend oder für einen Werkstattbereich spezifisch, soll Kritik und Weiterentwicklung zeitnah mit oder ohne Einberufung der Projektgruppe umgesetzt werden. Davon unabhängig werden zukünftig in der derzeit bestehenden Projektgruppe einmal jährlich alle Prozesse durchgegangen und besprochen, sodass diese aktuell bleiben und nicht aus den Augen verloren werden. « - Bernd Conrad, Geschäftsführer Lebenshilfe Detmold e. V.
»Ich beurteile die Prozessoptimierung als sehr gelungen und als wichtige Aufgabe. Es ist notwendig zu wissen, wer wie wofür verantwortlich ist, gerade auch beispielsweise bei der Einarbeitung neuer Fach- und Leitungskräfte. Bei der Vergegenwärtigung der Prozesse werden wir zum Nachdenken und zu Verbesserung angehalten. Wir diskutieren über die Arbeitsweise in der Werkstatt, wie es in der Vergangenheit noch nie geschehen ist. Dabei ist es natürlich für das Selbstbewusstsein wichtig, dass Prozesse abgeschlossen werden, was bei uns auch passiert. Nichts aussitzen und vergessen, sondern mitgestalten und verbessern - für alle Beteiligten. Auch das ist für uns ein Teil von Qualität. « Bernd Conrad, Geschäftsführer Lebenshilfe Detmold e. V.

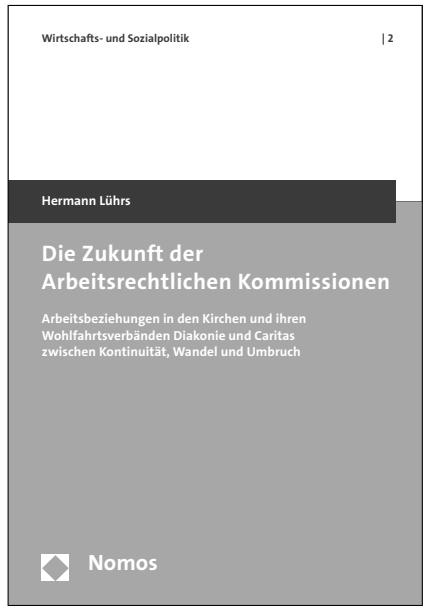

Die Zukunft der Arbeitsrechtlichen Kommissionen

Arbeitsbeziehungen in den Kirchen und ihren Wohlfahrtsverbänden Diakonie und Caritas zwischen Kontinuität, Wandel und Umbruch Von Dr. Hermann Lührs 2010, 279 S., brosch., 49, $-€$ ISBN 978-3-8329-5183-2

(Wirtschafts- und Sozialpolitik, Bd. 2)

Erstmals werden in dieser Studie die Regulierung der Arbeitsbedingungen und das spezielle System der Lohnfindung in Kirchen, Diakonie und Caritas sozialwissenschaftlich untersucht. Der Autor bietet einen umfassenden Überblick über Entstehung, Gegenwart und Zukunftsperspektiven der Arbeitgeber-/Arbeitnehmerbeziehungen in diesem Feld. Er rekonstruiert empirisch die kirchlichen Beschäftigungsverhältnisse seit 1920 und vergleicht die Form ihrer Regulierung mit dem Tarifvertragssystem.

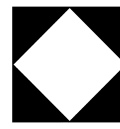

Nomos 